\title{
MRSA - Die Katastrophe droht
}

D eutschland hat's noch nicht kapiert. In Europa gibt es laut EARSS („European Antimicrobial Resistance Surveillance Study“) nur zwei Länder mit einem überdurchschnittlichen Anstieg methicillinresistenter Staphylococcus-aureus-Stämme (MRSA) - und das sind mit etwa 6\% pro Jahr England und Deutschland. Noch vor etwa 15 Jahren waren in Deutschland nur etwa $1 \%$ der Staphylococcus-aureus-Stämme methicillinresistent, jetzt sind wir bei ungefähr $20 \%$.

Viele Kliniker sagen, dies sei doch nicht so schlimm, in den USA liege der Prozentsatz bereits bei etwa $40 \%$ MRSA. Die Spanier, Italiener, Griechen, Portugiesen, Franzosen oder Belgier und auch wir Deutschen befänden uns damit noch in einem guten Mittelfeld. Doch diese Kliniker haben offensichtlich die neueste Literatur nicht gelesen: MRSA-Infektionen verlängern die Krankenhausverweildauer wesentlich mehr als methicillinsensible Staphylococcus-aureus-Infektionen, MRSAInfektionen sind mit einer höheren Mortalität verbunden, und MRSA-Infektionen sind wesentlich schwieriger zu behandeln - entweder mit toxischeren (z.B. Vancomycin) oder wesentlich teureren Antibiotika (Linezolid). Im Zeitalter der „diagnosis related groups“, der DRGs, wird so jede MRSA-Infektion durch die längere Verweildauer ein finanzielles Desaster.

Wieder einmal sind uns die Niederländer und Skandinavier weit voraus. Denn in diesen Ländern liegt die MRSA-Rate unter 1\%! Warum? Diese Länder beschränken den Gebrauch von Antibiotika in Klinik und Praxis („Restriktionspolitik“). Außerdem wird in den Niederlanden jeder Patient aus dem Ausland oder aus einer MRSA-Klinik zuerst gescreent und gleichzeitig isoliert. Erst wenn die Abstriche negativ sind, kommt er auf die Station („Search and Destroy-Politik“). In Deutschland dagegen fehlt bisher das kollektive Bewusstsein der Kliniker, dass MRSA-Infektionen unter allen Umständen vermieden werden sollten - und es fehlt eine nationale MRSA-Politik.

Was müssen wir auf jeden Fall machen? Zum einen ist jeder Patient mit einer MRSA-Infektion oder -Kolonisation sofort zu isolieren - auch wenn dafür ein Zweioder Dreibettzimmer kurzfristig in ein Einzelzimmer umgewandelt werden muss. Sind mehrere Patienten betroffen, können diese in ein Zimmer zusammengelegt werden (so genannte Kohortenisolierung). Zudem müssen wir in jeder Klinik sofort ein gezieltes Screeningprogramm implementieren.

Der Klinikumsvorstand des Universitätsklinikums Freiburg hat hierzu folgendes Vorgehen festgelegt: Bei der Aufnahme eines Patienten in die Intensivstation wird ein Nasenabstrich genommen bei Patienten, die älter sind als 60 Jahre (bei Prothesenträgern erfolgt zusätzlich ein Rachenabstrich), bei Patienten von anderen Kliniken, bei Patienten von anderen (externen) Intensivstationen, bei ausländischen Patienten, bei Patienten, die bereits seit längerem (länger als eine Woche) in der Klinik sind bzw. zum wiederholten Male aufgenommen werden, und bei chronischen Dialysepatienten. Zur Vereinfachung kann ein routinemäßiges Screening aller Intensivpatienten bei der Aufnahme sinnvoll sein.

Für alle anderen Stationen gilt: Frühere MRSA-Patienten (Nasenabstrich bilateral, Rachen- und Leistenabstrich, gegebenenfalls weitere Lokalisationen wie beispielsweise Wunden), Patienten mit chronischen Hautläsionen/Wunden (Nasenabstrich bilateral, Wunden/Dekubitus), Patienten aus Einrichtungen mit bekanntem MRSA-Problem (Nasenabstrich bilateral, Rachen- und Leistenabstrich, gegebenenfalls weitere Lokalisationen wie beispielsweise Wunden) und neu aufgenommene chronische Hämodialysepatienten werden bei der Aufnahme in die Station und dann im Abstand von sechs Monaten auf MRSA untersucht.

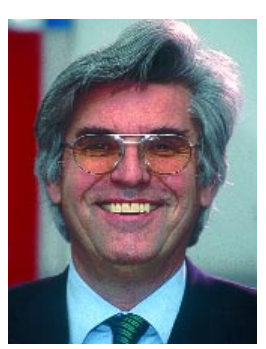

Prof. Dr. F. Daschner, Freiburg

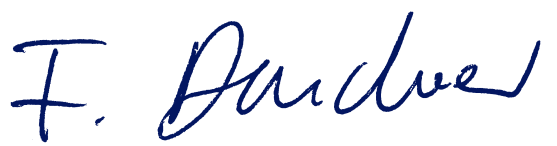

